\title{
Ocean Biogeochemistry and the Global Carbon Cycle: An Introduction to the U.S.S. Joint Global Ocean Flux Study
}

\author{
Ken O. Buesseler \\ Woods Hole Oceanographic Institution. Woods Hole, Massachusetts USA
}

In the early 1980s, ocean scientists were increasingly aware of the importance of biologically active elements, such as carbon, nitrogen and oxygen, in the regulation of climate and its effects on the habitability of the planet. As scientists reviewed details of the processes that control ocean carbon cycling and the links among oceanic, atmospheric and sedimentary carbon pools, it was clear that it was "hard to make the numbers add up" (Brewer et al., 1986). Fluxes of carbon into and out of the ocean were only crudely constrained, and little detail existed on the seasonal, regional and global patterns of carbon uptake and export or the flux of carbon between the ocean and its boundaries.

The U.S. Joint Global Ocean Flux Study (JGOFS), a component of the U.S. Global Change Research Program, grew out of the recommendations of a National Academy of Sciences workshop, held in Woods Hole in September 1984. The international program of which it is part was launched three years later under the auspices of the Scientific Committee on Oceanic Research. In 1989, as the first field studies were getting underway, JGOFS became a core project of the International Geosphere-Biosphere Programme.

A major ocean flux program was proposed in the U.S. that would include basin-scale process studies, long-term time-series programs and a global survey of carbon dioxide $\left(\mathrm{CO}_{2}\right)$ in the ocean (see JGOFS Goals below). Extrapolation of results to the global scale would be assisted by the largescale data sets emerging from satellite observations. Advances in numerical and ecological modeling and data assimilation would help us predict the global-scale response of oceanic biogeochemical processes to anthropogenic perturbations.

After more than a decade of scientific effort, the success of this study is clearly greater than the sum of its individual parts. With this special issue of Oceanography, we hope to highlight a few of the accomplishments of U.S. JGOFS. The authors were asked to bring to The Oceanography Society audience a synthesis of the changing paradigms of ocean biogeochemistry and its contribution to the global carbon cycle.

In this volume, you will see results from the time-series sites near Hawaii and Bermuda, from each of the four major process studies and from the global-scale survey of $\mathrm{CO}_{2}$, conducted in cooperation with the World Ocean Circulation Experiment. The modeling work discussed in this issue has contributed to advances in our understanding of the controls on upper ocean ecosystems and how these might change with future climate scenarios. An early policy of sharing data and rapid and open distribution via the internet has allowed all scientists access to a consistently high-quality database that will be a long-lasting legacy of U.S. JGOFS.

Advances in methods highlighted in this issue have led to some key breakthroughs in our ability to understand the dynamics of the dissolved organic carbon pools and particulate fluxes from the upper ocean to the depths. Common protocols and new chemical standards have allowed for accurate comparisons among studies. Within JGOFS and in the future, new technologies will lead to a better understanding of episodic and short-term shifts in ocean biogeochemistry and links among physical forcing, biological responses and chemical fluxes on a range of spatial scales.

As we think about the remaining challenges in ocean biogeochemistry, it is easy to forget how far we have come. After eight years' worth of days at sea, covering almost enough miles to circle the globe 16 times, considerable discussion and hundreds of papers, U.S. JGOFS scientists are confident that we have reduced the uncertainties in our understanding of the global ocean carbon cycle while gaining a new appreciation of the complexity of biogeochemical systems and their variability over time and space. This has been accomplished by many individuals with the help of some true community heroes and with steady support from the National Science Foundation in collaboration with the National Oceanic and Atmospheric Administration, the National Aeronautics and Space Administration, the Department of Energy and the Office of Naval Research. We are pleased to acknowledge the foresight of these agencies in supporting this work.

We appreciate the chance to present U.S. JGOFS through Oceanography to our fellow oceanographers and other interested scientists, educators and policymakers. A copy of our new U.S. JGOFS brochure, "A New Wave of Ocean Science," is included with this issue. Finally, as guest editor, I would like to thank the numerous U.S. JGOFS colleagues who contributed to the scientific insights you are reading about here, the authors of these articles and accompanying highlights, and the staff of the U.S. JGOFS Planning Office in Woods Hole, especially Mardi Bowles and Mary Zawoysky, for shepherding this issue to completion with assistance of the staff of Oceanography.

\section{Reference}

Brewer, P.G., K.W. Bruland, R.W. Eppley and J.J. McCarthy, 1986: The Global Ocean Flux Study (GOFS): Status of the U.S. GOFS Program. EOS, 67, 44.

\section{JGOFS Goals}

- to determine and understand on a global scale the processes controlling the time-varying fluxes of carbon and associated biogenic elements in the ocean, and to evaluate the related exchanges with the atmosphere, sea floor and continental boundaries;

- to develop a capacity to predict on a global scale the response of oceanic biogeochemical processes to anthropogenic pertur bations, in particular those related to climate change. 